\title{
Variable-Length Subcarrier Equalizers for Multicarrier Systems
}

\author{
Alexander M. Wyglinski \\ Peter Kabal \\ Fabrice Labeau \\ Department of Electrical \& Computer Engineering \\ McGill University, Montréal, Canada H3A 2A7 \\ email: $\{$ alexw, flabeau, kabal $\} @$ TSP.ECE.McGill.CA
}

\begin{abstract}
We present a novel algorithm for defining the lengths of subcarrier equalizers employed by wireless multicarrier transmission systems operating in frequency-selective fading channels. The equalizer lengths across the subcarriers are varied incrementally in a "greedy" fashion until the average mean squared error (MSE) is below some prescribed threshold. By varying the equalizer lengths, the overall complexity of the equalization is constrained while the system meets a minimum error performance. The results show that when a system employs variable-length equalizers defined by the proposed algorithm, it significantly outperforms a system employing constant-length equalizers of the same overall complexity.
\end{abstract}

Keywords: Multi-carrier modulation, equalization, adaptive loading

\section{Introduction}

Multicarrier modulation is widely deployed, including for high speed wireless local area networks [1,2]. Its primary advantage is that data can be transmitted on several parallel subcarriers simultaneously, each at a lower data rate. This effectively transforms the channel into a collection of subchannels. Therefore, instead of applying time domain equalization techniques to the channel, which could result in very long equalizers, short frequency domain equalizers can be applied to each subchannel to reverse the effects of the channel. The system employs synthesis and analysis filterbanks.

Previous studies have been investigating the use of persubcarrier frequency domain equalizers [3-5]. Our previous work $[6,7]$ was the first to use subcarrier equalizers of different lengths; the required lengths depend on the amount of distortion in the subcarriers. This distortion varies for different subcarriers given a frequency-selective fading channel.

We present a novel algorithm for determining the lengths of the subcarrier frequency domain equalizers. It improves upon our previous algorithm by choosing equalizer lengths that result in an overall MSE below some prescribed threshold, rather than an MSE threshold on individual subcarriers. The multicarrier framework is presented in Section 2 while Section 3 pro-

This research was partially funded by the Natural Sciences and Engineering Research Council of Canada (NSERC) and Le Fonds de Recherche sur la Nature et les Technologies du Québec.

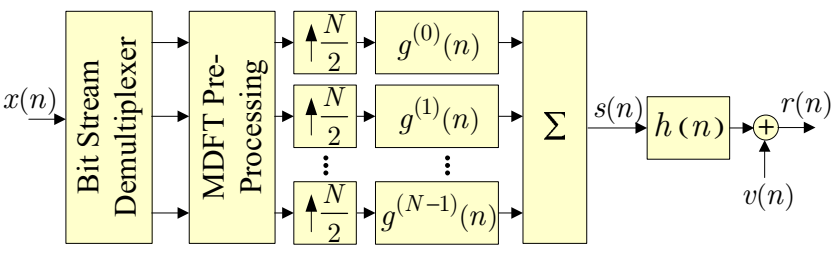

(a) Transmitter with channel

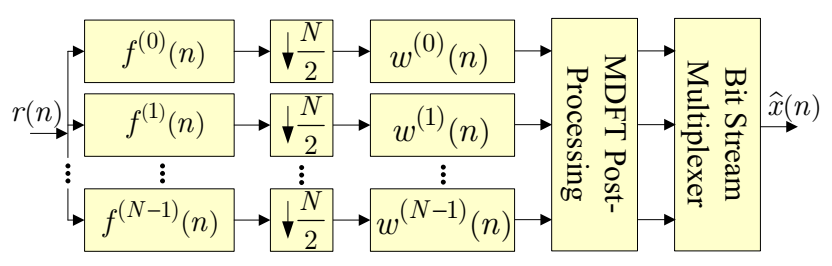

(b) Receiver with frequency domain equalizers

Fig. 1 Schematic of an MDFT multicarrier filterbank system

vides a detailed description of the proposed algorithm. The design of the subcarrier equalizers employed in this work is outlined in Section 4. Section 5 compares the results of employing our algorithm with a multicarrier system using constant-length subcarrier equalizers of equivalent overall complexity.

\section{Multicarrier Modulation}

In this work, we employ a modified discrete Fourier transform (MDFT) filterbank-based multicarrier system [5]. This type of multicarrier system was chosen for this work since the synthesis and analysis filters can be designed to be highly spectrally selective, and the intercarrier interference is minimized due to the phase offset between the real and imaginary data components. The general setup of this system is shown in Fig. 1. The high speed complex input symbol stream, $x(n)$, is demultiplexed into $N$ streams, with stream $i$ having $b_{i}$ bits per symbol epoch. The bit streams are modulated onto one of several signal constellations consisting of $M_{i}=2^{b_{i}}$ points. The outputs $x^{(i)}(n), i=0, \ldots, N-1$, are then MDFT preprocessed, where $x^{(i)}(n)$ is upsampled by a factor of 2 , the real and imaginary components are separated, one of the components is delayed by one sample, and the components are recombined. The MDFT pre-processing is shown in Fig. 2(a). The outputs $y^{(i)}(n), i=0, \ldots, N-1$, are upsampled by a factor $N / 2$ and filtered by synthesis filters $g^{(i)}(n), i=0, \ldots, N-1$, before being summed together, yielding the composite trans- 


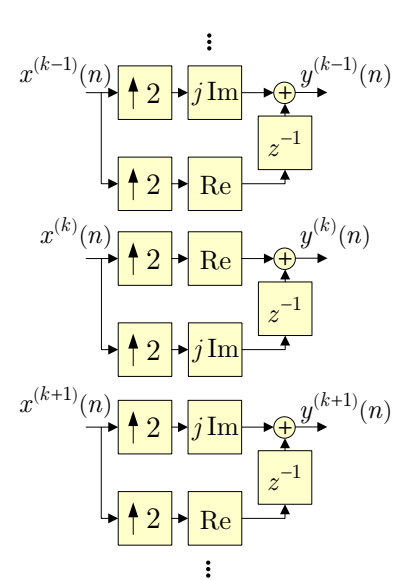

(a) MDFT pre-processing
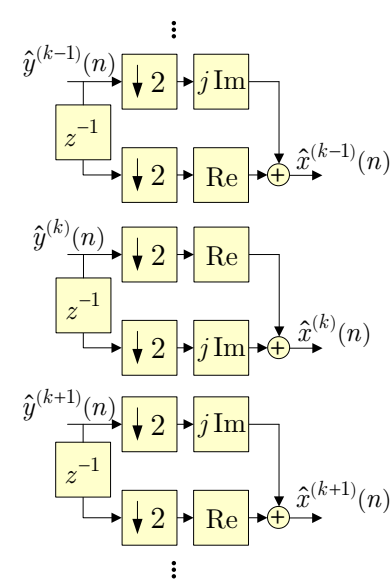

(b) MDFT post-processing
Fig. 2 Implementation of MDFT pre- and post-processing components

mit signal, $s(n)$. This signal is transmitted across the channel, where the multipath propagation and additive noise are modelled with channel impulse response $h(n)$ and noise $v(n)$. The received signal, $r(n)$, is separated into the $N$ subchannels using the analysis filters $f^{(i)}(n), i=0, \ldots, N-1$, downsampled by a factor $N / 2$, equalized using $w^{(i)}(n), i=0, \ldots, N-1$, MDFT post-processed (see Fig. 2(b)), demodulated, and then multiplexed together to form the estimate $\hat{x}(n)$.

\section{Proposed Algorithm}

Notation: Vectors are denoted by lower case, bold faced letters. Matrices are defined by upper case, bold faced letters. Scalars are denoted by lower case letters. The superscript in parentheses (if present) indicates the subcarrier while the subscript indicates either the sampling instants (vectors and scalars) or matrix dimensions (matrices, except for upsampling and downsampling matrices). The superscripts ${ }^{*},{ }^{T}$, and ${ }^{H} \mathrm{de}-$ note complex conjugation, transpose, and complex conjugation transpose, respectively.

\subsection{Algorithm Description}

The flow diagram of the proposed algorithm for obtaining the lengths of the subcarrier equalizers is shown in Fig. 3. Given a multicarrier system with $N$ subcarriers, there exists a subset of those subcarriers $S_{\text {data }}$ that are data-bearing while the remaining subcarriers are "turned off" (i.e. nulled). The algorithm begins by setting the lengths of the equalizers for the data-bearing subcarriers, $q^{(k)}, k \in S_{\text {data }}$, to a length of 1 . Note that all equalizer weights are computed using a closed-form MMSE expression. The equalizer weights $w^{(k)}(n)$ and corresponding theoretical MSE values $J^{(k)}$ for all the data-bearing subcarriers are computed given the lengths $q^{(k)}$. The mean of the $J^{(k)},\langle J\rangle$, is compared with the prescribed overall MSE threshold for the system, $J_{T}$. If $\langle J\rangle \leq J_{T}$, then none of the equalizer lengths needs to be increased in order to reduce $\langle J\rangle$ and the algorithm ends. However, if $\langle J\rangle>J_{T}$, then the al-

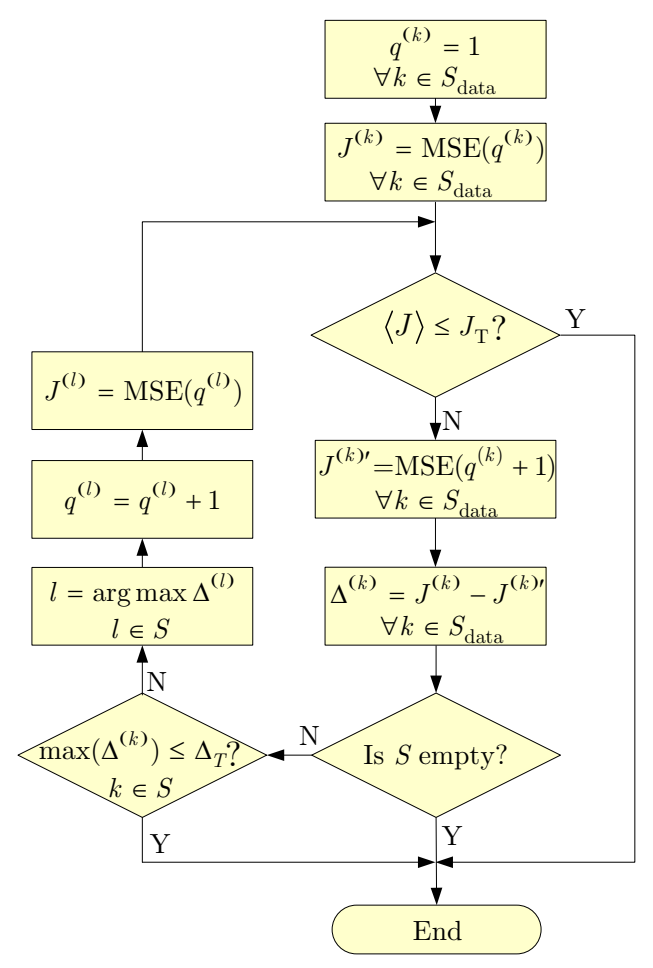

Fig. 3 Flow diagram of the adaptive subcarrier equalizer tap allocation algorithm $[6,7]$

gorithm needs to increase some of the lengths $q^{(k)}$ in order to satisfy $\langle J\rangle \leq J_{T}$.

This algorithm operates in a "greedy" fashion [8]. It incrementally increases the length of the subcarrier equalizer that maximizes the decrease in $\langle J\rangle$. Therefore, the algorithm computes the equalizer weights $w^{(k)^{\prime}}(n)$ and corresponding theoretical MSE values $J^{(k) \prime}$ when the lengths are $q^{(k)}+1$ for all data-bearing subcarriers. The differences $\Delta^{(k)}=J^{(k)}-J^{(k) \prime}$ are computed and the maximum difference is determined. We define the set $S$ as $S \subset S_{\text {data }}$ containing subcarriers with $q^{(k)} \leq q_{\max }$, where $q_{\max }$ is a prescribed subcarrier equalizer length limit. If $S$ is empty, the algorithm breaks out and ends. Otherwise $\Delta^{(k)}, k \in S$, is compared with the prescribed difference threshold $\Delta_{T}$. If $\max \left(\Delta^{(k)}\right) \leq \Delta_{T}$, then the algorithm breaks out and ends. If $\max \left(\Delta^{(k)}\right)>\Delta_{T}$, then the algorithm chooses subcarrier $l, l \in S$, which has the largest $\Delta^{(k)}$. The algorithm updates $q^{(l)}=q^{(l)}+1$ then computes $J^{(l)}$. Finally, $\langle J\rangle \leq J_{T}$ is compared and the process repeats.

\section{Equalizer Design}

\subsection{System Transfer Function}

The system transfer function of our MDFT filterbank multicarrier system is derived using a similar framework from our previous work [6, 7]. Referring to Figs. 1 and 2 as well as Section 2, the modulated data streams $x^{(i)}(n), i=0, \ldots, N-1$, are define for each subcarrier as a vector

$$
\mathbf{x}_{n, n-L+1}^{(k)}=\left[\begin{array}{lll}
x^{(k)}(n) & \cdots & x^{(k)}(n-L+1)
\end{array}\right]^{T},
$$


for $k=0, \ldots, N-1$ and of length $L$. These vectors are then used as inputs to the MDFT pre-processing stage of the system, the outputs of which are the vectors $\mathbf{y}_{n, n-2 L+1}^{(k)}, k=$ $0, \ldots, N-1$, with length $2 L$. These outputs are then upsampled using a $(2 L R+D) \times(2 L)$ upsampling matrix $\mathbf{T}_{u, R, D}$, defined as

$$
\left.\mathbf{T}_{u, R, D}=\left[\begin{array}{cccc}
0 & 0 & \cdots & 0 \\
\vdots & \vdots & \ddots & \vdots \\
0 & 0 & \cdots & 0 \\
1 & 0 & \cdots & 0 \\
0 & 0 & \cdots & 0 \\
\vdots & \vdots & \ddots & \vdots \\
0 & 0 & \cdots & 1
\end{array}\right]\right\} R+D-1
$$

where $R$ is the sampling rate, and $D$ is the delay. In this case, the sampling rate is $R=N / 2$ since combined with the MDFT pre-processing stage, which performs an upsampling by a factor of 2 , the overall upsampling rate is $N$ (i.e., critically-sampled). The delay is $D=2\lfloor\tau\rfloor+\left\lfloor\tau_{\mathrm{ch}}\right\rfloor$, where $\tau$ is the group delay of the synthesis or analysis filter, and $\tau_{\mathrm{ch}}$ is the group delay of the channel. By sufficiently zero-padding the transmitted vector, the analysis can adequately compensated for the effect of the total group delay introduced by the system prior to downsampling [9].

The upsampled signals are then filtered by the synthesis filters $\mathbf{g}_{0, P-1}^{(k)}, k=0, \ldots, N-1$, of length $P$. The filtered signals are then summed together and transmitted across the channel, with an impulse response $\mathbf{h}_{0, S-1}$ of length $S$. The received signal is decomposed into $N$ subcarriers using the analysis filters $\mathbf{f}_{0, P-1}^{(k)}, k=0, \ldots, N-1$, of length $P$ before being downsampled by the $\left(2 q^{(k)}\right) \times(2 L R+D-2 P-S+3)$ downsampling matrix $\mathbf{T}_{d, N / 2,0}=\mathbf{T}_{u, N / 2,0}^{T}$, where $2 q^{(k)}$ is the length of the fractionally-spaced MMSE equalizer, $\mathbf{w}_{0,2 q^{(k)}-1}^{(k)}$, $k=0, \ldots, N-1$.

Filtering is performed in this derivation using convolution matrices [6,7]. Therefore, we can represent $\mathbf{g}_{0, P-1}^{(k)}$ as an $(2 L R+D-P+1) \times(2 L R+D)$ convolution matrix

$$
\mathbf{G}^{(k)}=\left[\begin{array}{cccc}
\mathbf{g}_{0, P-1}^{(k) T} & 0 & \cdots & 0 \\
0 & \mathbf{g}_{0, P-1}^{(k) T} & \cdots & 0 \\
\vdots & \vdots & \ddots & \vdots \\
0 & 0 & \cdots & \mathbf{g}_{0, P-1}^{(k) T}
\end{array}\right]
$$

Furthermore, the channel $\mathbf{h}_{0, S-1}$ and the $k^{\text {th }}$ analysis filter $\mathbf{f}_{0, P-1}^{(k)}$, can be represented as $(2 L R+D-P-S+2) \times(2 L R+$ $D-P+1)$ and $(2 L R+D-2 P-S+3) \times(2 L R+D-P-S+2)$ convolution matrices, $\mathbf{H}$ and $\mathbf{F}^{(k)}$, respectively.

The transfer function corresponding to the $k^{\text {th }}$ subcarrier prior to the MDFT post-processing is

$$
\begin{aligned}
\overline{\mathbf{y}}_{n, n-2 q^{(k)}+1}^{(k)} & =\mathbf{T}_{d, R, 0} \mathbf{F}^{(k)} \mathbf{H} \sum_{l=0}^{N-1} \mathbf{G}^{(l)} \mathbf{T}_{u, R, D} \mathbf{y}_{n, n-2 L+1}^{(l)} \\
& +\mathbf{T}_{d, R, 0} \mathbf{F}^{(k)} \mathbf{v}_{0,2 L R+D-P-S+2}
\end{aligned}
$$

where $\mathbf{v}_{0,2 L R+D-P-S+2}$ is the additive white Gaussian noise (AWGN) contribution of the channel, of appropriate length.

To compensate for the distortion added to $\overline{\mathbf{y}}_{n, n-2 q^{(k)}+1}^{(k)}$ by the channel, as well as the synthesis and analysis filters, optimal $2 q^{(k)}$-tap fractionally-spaced MMSE equalizers $\mathbf{w}_{0,2 q^{(k)}-1}^{(k)}, k=0, \ldots, N-1$, are employed before performing MDFT post-processing. Thus, the equalized vector becomes

$$
\hat{y}^{(k)}(n)=\mathbf{w}_{0,2 q^{(k)}-1}^{(k) H} \overline{\mathbf{y}}_{n, n-2 q^{(k)}+1}^{(k)} .
$$

where the output is a scalar value at sampling instant $n$. In the next subsection, we present the derivation for the MMSE cost function at the output of the MDFT post-processing stage and optimal equalizer.

\subsection{Optimal MMSE Equalizer Derivation}

Since the desired real and imaginary information of $\overline{\mathbf{y}}_{n, n-2 q^{(k)}+1}^{(k)}$ are $90^{\circ}$ out-of-phase with each other and occur alternatively, we must consider reducing the distortion of the real and imaginary components separately at specific sampling instants.

Therefore, the mean squared error (MSE) cost function of the desired real and imaginary components can be defined as

$$
\begin{aligned}
& J^{(k)}=\frac{1}{2} E\left\{\left|\operatorname{Re}\left(y^{(k)}(2 m)-\hat{y}^{(k)}(2 m)\right)\right|^{2}\right\} \\
& +\frac{1}{2} E\left\{\left|\operatorname{Im}\left(y^{(k)}(2 m+1)-\hat{y}^{(k)}(2 m+1)\right)\right|^{2}\right\} \\
& =\frac{1}{8} E\left\{\mid y^{(k)}(2 m)-\hat{y}^{(k)}(2 m)\right. \\
& \left.+y^{(k) *}(2 m)-\left.\hat{y}^{(k) *}(2 m)\right|^{2}\right\} \\
& +\frac{1}{8} E\left\{\mid y^{(k)}(2 m+1)-\hat{y}^{(k)}(2 m+1)\right. \\
& \left.-y^{(k) *}(2 m+1)+\left.\hat{y}^{(k) *}(2 m+1)\right|^{2}\right\}
\end{aligned}
$$

where, without loss in generality, $n=2 m$ corresponds to the desired sampling instants for the real information in subcarrier $k$ while $n=2 m+1$ defines the desired sampling instants for the imaginary data.

Expanding Eq. (5) and employing Eq. (4) yields, after some algebra,

$$
\begin{aligned}
J^{(k)} & =\sigma_{y}^{2}-\operatorname{Re}\left\{\mathbf{p}_{y, r}^{(k) H} \mathbf{w}^{(k)}\right\}-\operatorname{Re}\left\{\mathbf{p}_{y, i}^{(k) H} \mathbf{w}^{(k)}\right\} \\
& +\frac{1}{4}\left(\operatorname{Re}\left\{\mathbf{w}^{(k) H} \mathbf{R}_{y, r}^{(k)} \mathbf{w}^{(k)}\right\}+\operatorname{Re}\left\{\mathbf{w}^{(k) H} \mathbf{R}_{y, i}^{(k)} \mathbf{w}^{(k)}\right\}\right. \\
& \left.+\operatorname{Re}\left\{\mathbf{w}^{(k) H} \mathbf{R}_{y, r}^{(k) \prime} \mathbf{w}^{(k) *}\right\}-\operatorname{Re}\left\{\mathbf{w}^{(k) H} \mathbf{R}_{y, i}^{(k) \prime} \mathbf{w}^{(k) *}\right\}\right)
\end{aligned}
$$


where

$$
\begin{aligned}
\mathbf{w}^{(k)} & =\mathbf{w}_{0,2 q^{(k)}-1}^{(k)} \\
\sigma_{y}^{2} & =E\left\{y^{(k)}(2 m) y^{(k) *}(2 m)\right\} \\
& =E\left\{y^{(k)}(2 m+1) y^{(k) *}(2 m+1)\right\} \\
\mathbf{p}_{y, r}^{(k) H} \mathbf{w}^{(k)} & =E\left\{y^{(k)}(2 m) \hat{y}^{(k) *}(2 m)\right\} \\
\mathbf{p}_{y, i}^{(k) H} \mathbf{w}^{(k)} & =E\left\{y^{(k)}(2 m+1) \hat{y}^{(k) *}(2 m+1)\right\} \\
\mathbf{w}^{(k) H} \mathbf{R}_{y, r}^{(k)} \mathbf{w}^{(k)} & =E\left\{\hat{y}^{(k)}(2 m) \hat{y}^{(k) *}(2 m)\right\} \\
\mathbf{w}^{(k) H} \mathbf{R}_{y, i}^{(k)} \mathbf{w}^{(k)} & =E\left\{\hat{y}^{(k)}(2 m+1) \hat{y}^{(k) *}(2 m+1)\right\} \\
\mathbf{w}^{(k) H} \mathbf{R}_{y, r}^{(k) \prime} \mathbf{w}^{(k) *} & =E\left\{\hat{y}^{(k)}(2 m) \hat{y}^{(k)}(2 m)\right\} \\
\mathbf{w}^{(k) H} \mathbf{R}_{y, i}^{(k) \prime} \mathbf{w}^{(k) *} & =E\left\{\hat{y}^{(k)}(2 m+1) \hat{y}^{(k)}(2 m+1)\right\}
\end{aligned}
$$

To get the minimum cost function for subcarrier $k, J_{\mathrm{min}}^{(k)}$, we need to find the optimal fractionally-spaced MMSE equalizer weights. To do that, we apply the Wirtinger derivative [10],

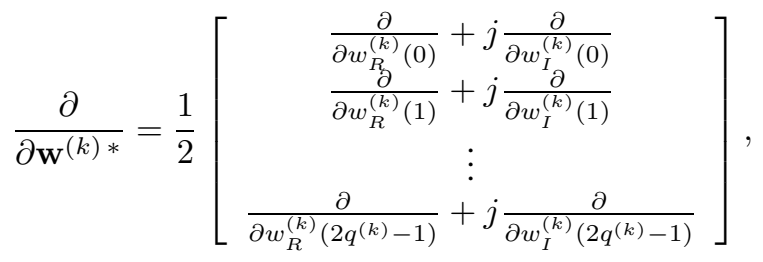

where $w_{R}^{(k)}(m)$ and $w_{I}^{(k)}(m)$ are the real and imaginary components of $w^{(k)}(m)$, to Eq. (6). This yields

$$
\begin{aligned}
\frac{\partial J^{(k)}}{\partial \mathbf{w}^{(k) *}}= & -4\left(\mathbf{p}_{y, r}^{(k)}+\mathbf{p}_{y, i}^{(k)}\right)+2\left(\mathbf{R}_{y, r}^{(k)}+\mathbf{R}_{y, i}^{(k)}\right) \mathbf{w}^{(k)} \\
& +2\left(\mathbf{R}_{y, r}^{(k) \prime}-\mathbf{R}_{y, i}^{(k) \prime}\right) \mathbf{w}^{(k) *} \\
= & 0
\end{aligned}
$$

which can be equivalently written as

$$
\begin{aligned}
2\left(\mathbf{p}_{y, r}^{(k)}+\mathbf{p}_{y, i}^{(k)}\right)= & \left(\mathbf{R}_{y, r}^{(k)}+\mathbf{R}_{y, i}^{(k)}\right) \mathbf{w}^{(k)} \\
& +\left(\mathbf{R}_{y, r}^{(k) \prime}-\mathbf{R}_{y, i}^{(k) \prime}\right) \mathbf{w}^{(k) *} .
\end{aligned}
$$

To solve for the optimal equalizer, we arrange Eq. (8) into a block matrix expression $\mathbf{A w}=2 \mathbf{p}$, namely

$$
\left[\begin{array}{ll}
\mathbf{A}_{11} & \mathbf{A}_{12} \\
\mathbf{A}_{21} & \mathbf{A}_{22}
\end{array}\right]\left[\begin{array}{l}
\operatorname{Re}\left(\mathbf{w}^{(k)}\right) \\
\operatorname{Im}\left(\mathbf{w}^{(k)}\right)
\end{array}\right]=2\left[\begin{array}{c}
\operatorname{Re}\left(\mathbf{p}_{y, r}^{(k)}+\mathbf{p}_{y, i}^{(k)}\right) \\
\operatorname{Im}\left(\mathbf{p}_{y, r}^{(k)}+\mathbf{p}_{y, i}^{(k)}\right)
\end{array}\right]
$$

where

$$
\begin{aligned}
& \mathbf{A}_{11}=\operatorname{Re}\left(\mathbf{R}_{y, r}^{(k)}+\mathbf{R}_{y, i}^{(k)}\right)+\operatorname{Re}\left(\mathbf{R}_{y, r}^{(k) \prime}-\mathbf{R}_{y, i}^{(k) \prime}\right) \\
& \mathbf{A}_{12}=-\operatorname{Im}\left(\mathbf{R}_{y, r}^{(k)}+\mathbf{R}_{y, i}^{(k)}\right)+\operatorname{Im}\left(\mathbf{R}_{y, r}^{(k) \prime}-\mathbf{R}_{y, i}^{(k) \prime}\right) \\
& \mathbf{A}_{21}=\operatorname{Im}\left(\mathbf{R}_{y, r}^{(k)}+\mathbf{R}_{y, i}^{(k)}\right)+\operatorname{Im}\left(\mathbf{R}_{y, r}^{(k) \prime}-\mathbf{R}_{y, i}^{(k) \prime}\right) \\
& \mathbf{A}_{22}=\operatorname{Re}\left(\mathbf{R}_{y, r}^{(k)}+\mathbf{R}_{y, i}^{(k)}\right)-\operatorname{Re}\left(\mathbf{R}_{y, r}^{(k) \prime}-\mathbf{R}_{y, i}^{(k) \prime}\right) .
\end{aligned}
$$

The optimal equalizer weights are then determined by solving $\mathbf{w}=2 \mathbf{A}^{-1} \mathbf{p}$, where the inverse of the block matrix $\mathbf{A}$ is [11]

$$
\mathbf{A}^{-1}=\left[\begin{array}{cc}
\mathbf{A}_{11}^{-1}+\mathbf{A}_{11}^{-1} \mathbf{A}_{12} \mathbf{S}^{-1} \mathbf{A}_{21} \mathbf{A}_{11}^{-1} & -\mathbf{A}_{11}^{-1} \mathbf{A}_{12} \mathbf{S}^{-1} \\
-\mathbf{S}^{-1} \mathbf{A}_{21} \mathbf{A}_{11}^{-1} & \mathbf{S}^{-1}
\end{array}\right]
$$

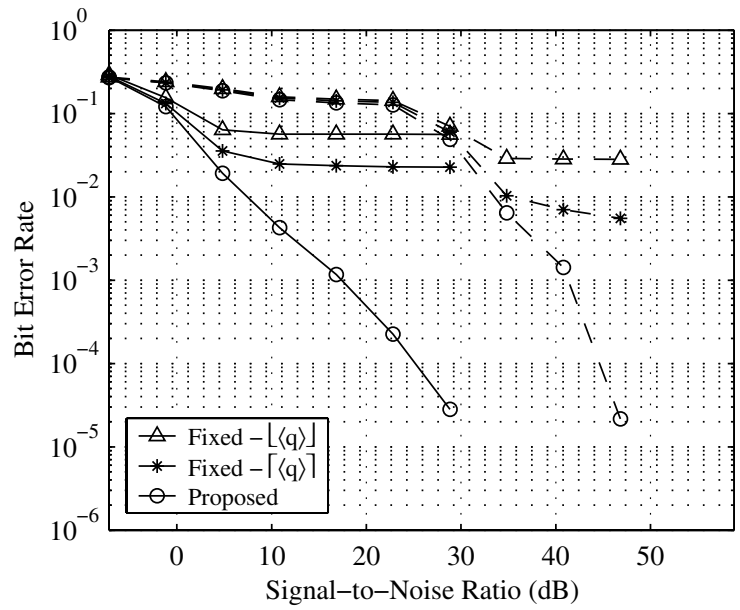

Fig. 4 BER performance for 8 subcarriers with BPSK and 16-QAM modulation when adaptive subcarrier equalizer tap allocation is employed along with fixed length equalizers of equivalent complexity.

and $\mathbf{S}=\mathbf{A}_{22}-\mathbf{A}_{21} \mathbf{A}_{11}^{-1} \mathbf{A}_{12}$ is its Schur complement.

Note that the value of $q^{(k)}$ is determined by the proposed algorithm of Section 3.

\section{Results}

Using the ETSI HiperLAN/2 indoor channel models [12], we employ the proposed algorithm in an MDFT filterbank multicarrier system [5]. The synthesis and analysis filters are modulated versions of a root raised cosine lowpass filter. Many of the operating parameters of the system correspond to the IEEE Std. 802.11a [1], including the modulation schemes (BPSK, QPSK, rectangular 16-QAM, and rectangular 64-QAM modulation), subcarrier spacing, and operating frequency $(5 \mathrm{GHz})$. Since the system is operating in an indoor environment, the channel is quasi-stationary. Therefore, the channel is assumed to be time-invariant over a sufficiently long period of time and that the equalizer weights and lengths need to be determined once for a specific channel. Moreover, we assume the channel is perfectly known at the receiver, although in practice dataaided channel estimation techniques would be employed.

Figs. 4 and 5 show the bit error rate (BER) performance of a MDFT filterbank multicarrier system, operating in an ETSI HiperLAN/2 Channel A, employing the proposed algorithm for an 8-subcarrier portion of a 52-subcarrier system. The average length of the equalizers range from $\langle q\rangle=1.25$ taps per subcarrier (BPSK at $7 \mathrm{~dB}$ ) to $\langle q\rangle=2.875$ taps per subcarrier (64-QAM at $52 \mathrm{~dB}$ ). However, the majority of taps in this case were used by the equalizer of the $5^{\text {th }}$ subcarrier since it was located in a spectral valley. For instance, in the BPSK case, all the equalizers used 1 tap, except for the $5^{\text {th }}$ subcarrier, which used 3 taps. To determine the benefits of variablelength subcarrier equalizers and the proposed algorithm, we compare their impact on BER performance with systems employing constant-length subcarrier equalizers having lengths equal to $\lfloor\langle q\rangle\rfloor$ and $\lceil\langle q\rangle\rceil$. This is to ensure a fair comparison 


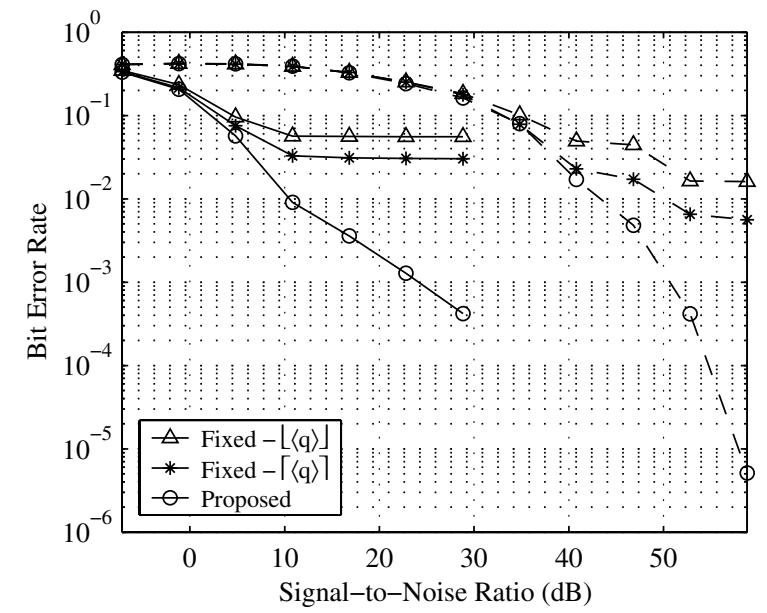

Fig. 5 BER performance for 8 subcarriers with QPSK and 64-QAM modulation when adaptive subcarrier equalizer tap allocation is employed along with fixed length equalizers of equivalent complexity.
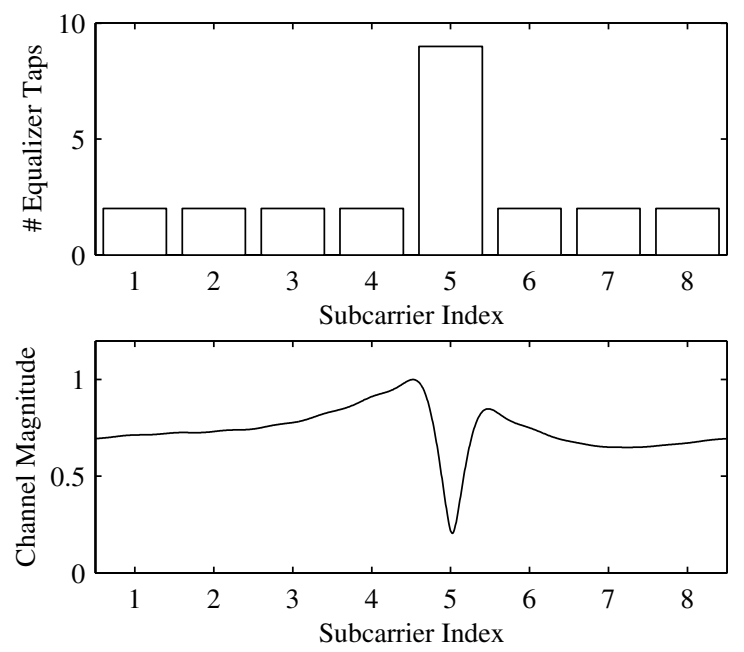

Fig. 6 Relationship between the subcarrier equalizer tap allocation and the channel frequency (magnitude) response.

by making the overall complexity of the equalizers equivalent.

The results show that compared to the systems employing subcarrier equalizers of constant lengths, the system employing our proposed algorithm outperforms them in BER. For instance, in Fig. 4, the difference at a signal-to-noise ratio (SNR) of $23 \mathrm{~dB}$ for BPSK modulation is two orders of magnitude. The same is true for the other modulation schemes (QPSK at an SNR of $29 \mathrm{~dB}, 16-\mathrm{QAM}$ at an SNR of $46 \mathrm{~dB}$, and 64-QAM at an SNR of $58 \mathrm{~dB}$ ). This is due to the fact that several poorly performing subcarriers do not employ an equalizer of a sufficient length. As a result, the error due to this subcarrier dominates the overall error performance of the system. However, with the proposed algorithm, each subcarrier uses an equalizer which has a sufficient length.

\section{Conclusion}

In this paper, we present a novel algorithm for defining the lengths of the subcarrier frequency domain equalizers. It is shown that by tailoring the lengths of the subcarrier equalizers to the channel conditions using the proposed algorithm, the system outperforms other systems employing constant-length subcarrier equalizers of equivalent overall complexity, in terms of error robustness.

\section{References}

[1] IEEE Std. 802.11a, "Wireless LAN medium access control (MAC) and physical layer (PHY) specifications: High-speed physical layer in the $5 \mathrm{GHz}$ band," Nov. 1999.

[2] ETSI TS 101 475, "Broadband radio access networks (BRAN): HIPERLAN type 2; physical (PHY) layer,” Dec. 2001.

[3] J. Louveaux, Filter Bank Based Multicarrier Modulation for $x$ DSL Transmission. PhD thesis, Université Catholique de Louvain, Louvain-la-Neuve, Belgium, May 2000.

[4] K. Van Acker, G. Leus, M. Moonen, O. Van de Wiel, and T. Pollet, "Per tone equalization for DMT-based systems," IEEE Trans. Commun., vol. 49, pp. 109-119, Jan. 2001.

[5] T. Wiegand and N. J. Fliege, "Equalizers for transmultiplexers in orthogonal multiple carrier data transmission," Proc. Euro. Signal Processing Conf., vol. 2, pp. 1211-1214, 1996.

[6] A. M. Wyglinski, P. Kabal, and F. Labeau, "Adaptive filterbank multicarrier wireless systems for indoor environments," Proc. 56th IEEE Veh. Technol. Conf. - Fall, pp. 336-340, Sept. 2002.

[7] A. M. Wyglinski, P. Kabal, and F. Labeau, "Adaptive bit and power allocation for indoor wireless multicarrier systems," Proc. Int. Conf. Wireless Commun., pp. 500-508, July 2003.

[8] A. Gersho and R. M. Gray, Vector Quantization and Signal Compression. Kluwer Academic Publishers, 1992.

[9] T. Pollet and M. Peeters, "Synchronization with DMT modulation," IEEE Commun. Mag., pp. 80-86, Apr. 1999.

[10] S. Haykin, Adaptive Filter Theory. Prentice Hall Information and System Sciences Series, Prentice Hall, 3rd ed., 1996.

[11] T. K. Moon and W. C. Stirling, Mathematical Methods and Algorithms in Signal Processing. Prentice-Hall, 2000.

[12] J. Medbo and P. Schramm, "Channel models for HIPERLAN/2 in different indoor scenarios," ETSI EP BRAN Doc. No. 3ERI085B, European Telecommunications Standards Institute, 1998. 